\title{
Diagnostic Accuracy of FNAC in Evaluation of Breast Lumps
}

Authors

\section{Sumeera Farhath SK ${ }^{1}$, K.Sravan Kumar ${ }^{2}$ Rita Singh $^{3}$, V. Ashok Kumar}

${ }^{1,2}$ Postgraduate, Dept of General Surgery, MNR Medical College and Hospital, Sangareddy, Telangana

${ }^{1}$ Email: sumeerafarhath@gmail.com

${ }^{3}$ Assistant Professor, Dept of General Surgery, MNR Medical College and Hospital, Sangareddy, Telangana ${ }^{4}$ Professor and Head, Dept of General Surgery, MNR Medical College and Hospital, Sangareddy, Telangana

Email:vashokk11@gmail.com

\section{ABSTRACT}

Background and objectives: This study was conducted to compare the diagnostic accuracy of fine needle aspiration cytology (FNAC)in differentiating the benign and malignant lesions of palpable breast lump with histopathological correlation.

Methods: This study was conducted at MNR MEDICAL COLLEGE AND HOSPITAL, Sangareddy, Telangana. The study included 50 patients who presented with palpable breast lumps to the surgical out-patient department. Thorough physical examination was done and the patients were subjected to fine needle aspiration cytology of the palpable breast lump. The cytological examination was classified as:

Insufficient sample - C1, Benign - C2, Probably Benign - C3,

Suspicious of malignancy - C4, Malignant - C5.All these patients were subjected to open/excision biopsy followed by histopathological examination. The diagnostic accuracy of the fine-needle aspiration was then compared with the gold standard histopathology.

Results: Most of the cases were of benign pathology of which Fibroadenoma were most common (64\%). It was seen that all the patients were female patients in the age group of 15-75 years. Majority of the lumps were found to be in the upper outer quadrant (46\%).

The sensitivity of fine needle aspiration cytology was found to be $83.33 \%$,

The specificity $100 \%$, Positive predictive value $100 \%$ and Negative predictive value $97.77 \%$.

Conclusion: In patients with a palpable breast lump fine needle aspiration cytology can help to rule out a malignant lesion. This can hence afford as a cost-effective method in diagnosing breast lesions. Also due to its good tolerability and high diagnostic accuracy the patient need not be subjected to further evaluation which helps in the psychological well being of the patient.

But, FNAC results when negative or suspicious should be correlated with clinical examination and imaging findings to prevent false positives and false negatives. A diagnosis suspicious for malignancy should be confirmed by open biopsy.

Keywords- Breast cancer, Breast lumps, Cytology, Diagnostic accuracy, Fine needle aspiration cytology, Histopathology 


\section{INTRODUCTION}

Breast is a dynamic structure, which undergoes changes throughout women's reproductive life, and superimposed on this, cyclical changes throughout the menstrual cycle. The pathogenesis involves disturbance in the breast physiology extending from an extreme normality to well defined disease process. ${ }^{[1]}$

Breast lump is the clinical presentation of numerous breast diseases ranging from innocent benign cysts to malignant lesions. Distinction of benign from malignant is of paramount importance for patient care and proper management. ${ }^{[2]}$

Donegan ${ }^{[3]}$ stated that most breast cancers appear as palpable masses, usually found by patients. However not all palpable abnormalities represent discrete masses. This is especially true in women younger than 40 years of age, in whom normal glandular nodularity may be mistaken for dominant masses.

Breast cancer is the most common site specific cancer in women and is the leading cause of death from cancer for women of age 40 to 44 year ${ }^{[4,5]} \mathrm{It}$ accounts for $33 \%$ of all female cancers and is responsible for $20 \%$ of the cancer related deaths in women $^{[5]}$

However, it is seen that misdiagnosed breast cancer accounts for the greatest number of malpractice claims for errors in diagnosis. ${ }^{[5]}$

The first step to evaluate a case of breast lump is by clinical assessment. Although a clinician can confidently make the diagnosis of either benign or malignant lesion, but there is always a possibility of error.

So this emphasises the need for an investigation which has a good diagnostic accuracy and patient compliance.

Presently a wide range of diagnostic modalities are available for the evaluation of breast lump. Conventional open biopsy, considered to be the gold standard for confirming diagnosis, has significant morbidity, is costly and time consuming. Hence, Fine needle aspiration cytology with its good patient acceptance and tolerability has been chosen for this study to evaluate its diagnostic accuracy.

\section{MATERIAL AND METHODS}

A study was conducted to compare the results of fine needle aspiration cytology with histopathology in the evaluation of breast lumps. This study was conducted at MNR MEDICAL COLLEGE, Sangareddy, Telangana for a duration of 1 year in the year 2014.The study included 50 patients who presented with palpable breast lumps to the surgical out-patient department selected by simple random sampling. Lumps associated with fungation of skin, etc. and patients who underwent prior biopsy were excluded from the study .Thorough physical examination was done and the details were entered in a case proforma after obtaining a valid informed consent. The patients were then subjected to fine needle aspiration cytology of the palpable breast lump. The aspirate was expelled on to a clean glass slide and fixed with $90 \%$ ethanol. Staining was done with Papanicolaou or Hematoxylin -Eosin and subjected to microscopic examination. The cytological reporting was classified as:

Insufficient sample - $\mathrm{C} 1$, Benign - C2,Probably Benign - C3, Suspicious of malignancy - C4, Malignant - C5.All these patients were subjected to open/excision biopsy followed by histopathological examination. The diagnostic accuracy of the fineneedle aspiration was then compared with the gold standard histopathology. The results were documented and interpreted.

\section{RESULTS}

Table 1: Distribution of breast neoplasm according to age

\begin{tabular}{|l|l|l|}
\hline Age group & No. of cases & Percentage \% \\
\hline $15-20$ & 12 & 24 \\
\hline $21-30$ & 12 & 24 \\
\hline $31-40$ & 13 & 26 \\
\hline $41-50$ & 7 & 14 \\
\hline $51-60$ & 5 & 10 \\
\hline $61-75$ & 1 & 2 \\
\hline
\end{tabular}


Table 2: Distribution of breast neoplasms according to the side of involved breast

\begin{tabular}{|l|l|l|}
\hline Side & No of cases & Percentage \% \\
\hline Right breast & 28 & 56 \\
\hline Left breast & 22 & 44 \\
\hline Bilateral & - & - \\
\hline
\end{tabular}

Table 3: Distribution according to the site of lump

\begin{tabular}{|l|l|l|}
\hline Quadrant & No of cases & Percentage \% \\
\hline Upper outer & 23 & 46 \\
\hline Upper inner & 10 & 20 \\
\hline Lower inner & 6 & 12 \\
\hline Lower outer & 3 & 6 \\
\hline Central & 5 & 10 \\
\hline Axillary tail & 3 & 6 \\
\hline
\end{tabular}

Table 4: Distribution according to FNAC diagnoses

\begin{tabular}{|l|l|l|}
\hline FNAC diagnosis & No. of cases & Percentage \% \\
\hline C1 & - & - \\
\hline C2 & 40 & 80 \\
\hline C3 4 & 6 & 12 \\
\hline C4 & - & - \\
\hline C5 & 4 & 8 \\
\hline
\end{tabular}

Table 5: Distribution according to histopathological examination

\begin{tabular}{|l|l|l|}
\hline $\begin{array}{l}\text { Histopathological } \\
\text { diagnosis }\end{array}$ & $\begin{array}{l}\text { Number of } \\
\text { cases }\end{array}$ & Percentage\% \\
\hline Fibroadenoma & 32 & 64 \\
\hline Fibrocystic disease & 10 & 20 \\
\hline Benign Phyllodes & 2 & 4 \\
\hline $\begin{array}{l}\text { Ductal carcinoma in } \\
\text { situ }\end{array}$ & 2 & 4 \\
\hline Invasive carcinoma & 4 & 8 \\
\hline Total & 50 & 100 \\
\hline
\end{tabular}

Table 6: Distribution of benign and malignant cases on FNAC

\begin{tabular}{|l|l|l|}
\hline Lesions & Number of Cases & Percentage $\%$ \\
\hline Benign & 46 & 92 \\
\hline Malignant & 4 & 8 \\
\hline
\end{tabular}

Table 7: Distribution of benign and malignant cases on histopathology

\begin{tabular}{|l|l|l|}
\hline Lesions & No of Cases & Percentage \% \\
\hline Benign & 44 & 88 \\
\hline Malignant & 6 & 12 \\
\hline
\end{tabular}

Table 8: Comparison of FNAC with Histopatholog

\begin{tabular}{|l|l|l|l|}
\hline \multirow{2}{*}{ FNAC } & \multicolumn{2}{|c|}{ Histopathology } & \multirow{2}{*}{ Total } \\
\cline { 2 - 3 } & Malignant & Benign & 5 \\
\hline Malignant & 5 & - & 45 \\
\hline Benign & 1 & 44 & 50 \\
\hline Total & 6 & 44 & \\
\hline
\end{tabular}

Specificity- $100 \%$

Sensitivity- $83.33 \%$

Positive predictive value- $100 \%$

Negative predictive value- $97.77 \%$

\section{DISCUSSION}

In this study 50 patients presenting with the complaint of a breast lump were included. All the patients presenting with the complaint of lump in breast were females. In a study carried out in Nigeria by Babatunde A Ayoade etal ${ }^{[6]}$ they had a similar age group to our study. The age range was 14-70 years. The commonest symptom was breast lump in $111(91.7 \%)$ patients. In that study out of the 121 patients, 119 were females, two were males. It was noted that majority of the patients about 26 $\%$ were in the age group of 31-40 years of age. Most of the lumps were not associated with any other symptoms. However, $10 \%$ were associated with pain, $4 \%$ associated with discharge from nipple. The average size of the lumps was around 3-4 cm. Majority of the patients presented with lump in upper outer quadrant 46\%. All the cases had unilateral disease. Only 2 cases presented with multiple lumps rest of them presented with a single lump.

All the lumps were subjected to Fine needle aspiration and cytology. The results were classified as

C1-insufficient sample, C2- benign lesions, C3probably benign, $\mathrm{C} 4$ - suspicious of malignancy and C5- malignant .On staining, cytology and reporting, it was seen that about $80 \%$ comprising of 40 cases were diagnosed as C2 (benign lesions), C3 -12\% (probably benign) and C5 about $8 \%$ ( malignant). Initially a few cases reported as

C1 (insufficient sample) were repeated again and hence there were no cases with $\mathrm{C} 1$ score. Of all the lesions it was noted that majority of them are of benign origin about $92 \%$ and $8 \%$ malignant. All these lumps were subjected to excision and histopathological examination which revealed about $88 \%$ of benign pathology and $12 \%$ malignant pathology. It included $64 \%$ of fibroadenomas , $20 \%$ 
fibrocystic disease, $4 \%$ diagnosed as ductal carcinoma in situ and $8 \%$ invasive carcinoma.

The most common benign pathology was seen to be a fibroadenoma. Fibroadenoma is the most common benign breast mass. Although most breast cancers occur in women older than 50 years, 31 percent of women diagnosed with breast cancer younger than 50 years. $^{[7]}$

2 cases which were diagnosed on FNAC as probably benign turned out to be ductal carcinoma in situ on histopathology. So by comparing the results of FNAC with that of histopathology the diagnostic accuracy of FNAC was assessed.

It was done by finding the sensitivity, specificity, negative predictive value and the positive predictive value which were $83.33 \%, 100 \%, 97.77 \%$ and $100 \%$ respectively in this study.

It is seen that the sensitivity ranges from $80-95 \%{ }^{[8]}$ and false positive aspirates are seen in less than $1 \%$ of cases. False negative results are seen in $4-10 \%$ and are most common in fibrotic or well differentiated tumours ${ }^{[8]}$. Our results are comparable to this study.

FNAC is highly sensitive (98 to $99 \%$ ) and has a positive predictive value of 99 percent and a negative predictive value of 86 to 99 percent. ${ }^{[7,9]}$

In a study done by Ahmed I et al ${ }^{[10]}$, they concluded that in non-concordant cases, FNAC stands as single most important investigation. However, due to its false negative results, other components of triple test need to be employed to enhance its efficacy and diagnostic yield.

Reinikainen et al ${ }^{[11]}$ had a series of 84 patients where they compared Mammogram, USG, FNAC, HPE by a scoring system. They have found sensitivity and specificity of FNAC to be $92 \%$ and $83 \%$ respectively.

In a study done by Philip J Drew et al ${ }^{[12]}$, sensitivity of fine-needle aspiration cytology was $79.1 \%$ and specificity of $97 \%$.the specificity is similar to our study.

Al-Mulhim et al ${ }^{[13]}$, in a study to assess accuracy of the "triple test" found that fine-needle aspiration cytology (FNAC) showed 91.7\% sensitivity, 100\% specificity and $100 \%$ positive predictive value.
In 1998, J.R.C Sainsbury et al ${ }^{[14]}$ conducted a prospective study on 112 patients with clinically discrete benign breast lumps. Diagnosis was achieved by clinical examination and FNAC. $68 \%$ of patients experienced resolution of their lumps over a period of 2 years. Resolution of both fibroadenoma and discrete areas of fibroadenosis was observed. Hence, with a good diagnostic accuracy patients diagnosed as benign lesions of FNAC can be treated by observation and medical management without subjecting to excision biopsy.

\section{CONCLUSION}

FNAC can be used as a primary pre operative diagnostic modality. It is less painful, produces speedy results and is inexpensive. It can be easily applied to clinically palpable lumps. It is a less demanding technique, has minimal complications, and suits for OPD procedure. It can be easily repeated and it can be employed in multiple lesions. In patients with a palpable breast lump fine needle aspiration cytology can help to rule out a malignant lesion. This can hence afford as a cost -effective method in diagnosing breast lesions. Also due to its good tolerability and high diagnostic accuracy the patient need not be subjected to further evaluation which helps in the psychological well being of the patient.

But, FNAC results when negative or suspicious should be correlated with clinical examination and imaging findings to prevent false positives and false negatives. A diagnosis suspicious for malignancy should be confirmed by open biopsy.

\section{REFERENCES}

1. Sainsburg RC. The breast. In:Rusell RCG, Williams NS, Bulsrode CJK. editors. Bailey and Love's Short Practice of Surgery. $24^{\text {th }}$ edition, Arnold,London;2004: p824-846.

2. Ibrar Ahmed, RashedNazir, M.Y.Chaudry, Saddia Kundi. Triple assessment of Breast lump. JCPSP 2007, vol.17(9):535-8

3. Donegan WL. Evaluation of a palpable breast mass. N Engl J Med 1992;327:937-42 
4. Baum M. Carcinoma of the Breast. InRecent advances in surgery, London. Churchill Livingston, 1984;241:58

5. Kirby I.Bland, Samuel W .Beenken, Edward M.CopelandIII. TheBreast.In-Schwartz's Principles of Surgery, Charles F. Brunicardi, McGrawHill 2005, P.470,475-77

6. Babatunde A Ayoade, Adedayo O Tade, and Babatunde A Salami. Clinical Features and Pattern of Presentation of Breast Diseases in Surgical Outpatient Clinic of a Suburban Tertiary Hospital in South-West Nigeria .Niger J Surg. 2012 Jan-Jun; 18(1): 13-16.

7. Klein S. Evaluation of Palpable Breast Masses. Am FamPhys2005; 71(9):324-343.

8. Harold J.Burstein, Jay R. Harris, Monica Morrow. Malignant tumors of Breast. InDevita, Hellman, Rosenerg' scancer: Principles and Practice of oncology. Lippincott 2008,8th ed:P.1612

9. Lindholm K. Breast. In: Orell SR, Sterrett GF, Whitaker D, eds. Fine NeedleAspiration Cytology. 4th ed. India: Churchill Livingstone; 2005: p 165-226.

10. Ahmed I, Nazir R, Chaudhary MY, Kundi S. Triple assessment of breastLump; J Coll Physicians Surg Pak. 2007 Sep; 17(9):535-8.

11. Reinikainen HT, Rissanen TJ, Pilippo UK, Paivansalo MJ; Contribution of ultrasonography and fine-needle aspiration cytology to the differentialdiagnosis of palpable solid breast lesions. Acta Radio 1999 Jul;40(4):383-9

12. Philip J Drew, Lindsay W Turnbull, Sumohan Chatterjee, John Read, Peter J Carleton, et al. Prospective Comparison of Standard Triple Assessment and Dynamic Magnetic Resonance Imaging of the Breast for the Evaluation of Symptomatic Breast Lesions. Annals of Surgery1999; 230(5) :680-5

13. Al-Mulhim AS, Sultan M, Al-Mulhim FM, Al-Wehedy A, Ali M, Al-Suwaigh A, AlDhafiri S, Bavemen O. Accuracy of the triple test in thediagnosis of palpable breast masses in Saudi females. Ann Saudi Med 2003 May-Jul;23(3-4):158-61

14. J.R.C.Sainsbury,S.Nicholson,G.K.Needham, V.Wadehra,J.R Farndon.Natural history of benign breast lump. Br J Surg1988; 75 : $1080-1082$. 